\title{
Assessment of Neuropathic Pain in Ankylosing Spondylitis: Prevalence and Characteristics
}

Ling Zhou $\cdot$ Ting Li $\cdot$ Xin $\mathrm{Wu} \cdot$ Hongjuan $\mathrm{Lu} \cdot \mathrm{Li}$ Lin $\cdot$ Lingying Ye $\cdot$ Jian Yin $\cdot$ Juan Zhao

Xiuwen Wang $\cdot$ Jianye Bian $\cdot$ Huji Xu (1)

Received: June 30, 2021 / Accepted: August 13, 2021 / Published online: September 4, 2021

(c) The Author(s) 2021

\section{ABSTRACT}

Introduction: Pain in ankylosing spondylitis is currently considered an inflammatory pain (IP). However, it was found that some patients still had the sensation of pain even without inflammation. Our study was to investigate the prevalence and characteristics of neuropathic pain (NeP) in Chinese Han ankylosing spondylitis (AS) patients.

Methods: The study consisted of three parts. Firstly, we assessed the prevalence and clinical data of NeP in 182 AS patients. Secondly, we evaluated pain improvement after etanercept therapy in 63 patients. Finally, serum neurotransmitters were measured for 20 AS patients and ten healthy controls (HC).

Ling Zhou and Ting Li contributed equally to this work.

L. Zhou · T. Li - X. Wu · H. Lu - L. Lin · L. Ye .

J. Yin $\cdot$ J. Zhao $\cdot$ X. Wang $\cdot$ J. Bian $\cdot H$. Xu $(\bowtie)$

Department of Rheumatology and Immunology,

Shanghai Changzheng Hospital, Naval Medical

University, 415 Fengyang Road, Shanghai 200003,

China

e-mail: xuhuji@smmu.edu.cn

H. $\mathrm{Xu}$

Peking-Tsinghua Center for Life Sciences, Tsinghua

University, Beijing 100084, China

H. Xu

School of Clinical Medicine, Tsinghua University,

Beijing 100084, China
Results: Out of 182 AS patients, our study showed that 14 patients (7.70\%) had likely NeP and 55 (30.21\%) had uncertain NeP. There were significant differences among the three groups with respect to nocturnal pain (NP), peripheral pain (PP), total back pain (TBP), BASDAI, ASDAS-CRP, HAD-A, HAD-D, and BASDAIfatigue except fort CRP concentrations. Principal component analysis (PCA) of AS pain revealed that the weight of NeP was greater than $\mathrm{PP}$ in the first principal component (0.703 vs. 0.639). Structural equation modeling (SEM) revealed that NeP altered disease activity $(\beta=0.62$, $P<0.001)$, which influenced psychological status $(\beta=0.42, P<0.001)$. Of 63 patients who used etanercept for 3 months, significant improvement was found in NP, TBP, and PP (all $P<0.0001)$ but not in PDQ $(10.60 \pm 6.85$ vs. $9.98 \pm 6.40, \quad P=0.0671)$. Serum norepinephrine concentrations in patients with PDQ $>19$ were higher than those in patients with PDQ $\leq 19$ and HC.

Conclusions: We conclude that $\mathrm{NeP}$ contributes to pain in AS patients.

Keywords: Ankylosing spondylitis; Neuropathic pain; Neurotransmitters 


\section{Key Summary Points}

Why carry out this study?

Pain in ankylosing spondylitis (AS) is currently considered an inflammatory pain (IP). However, it was found that some patients still had the sensation of pain even without inflammation.

Our study was to evaluate the prevalence and characteristics of neuropathic pain (NeP) in AS patients, and to investigate the difference between $\mathrm{NeP}$ and IP, especially in the outcome of anti-TNF treatment and the serum concentration of neurotransmitters.

\section{What was learned from the study?}

Our study showed that $37.91 \%$ of AS patients had likely or uncertain NeP. Principal component analysis (PCA) of AS pain revealed that the weight of $\mathrm{NeP}$ was greater than peripheral pain (PP) in the first principal component (0.703 vs. 0.639). pain-DETECT Questionnaire (PDQ) was not significantly improved after etanercept treatment $(10.60 \pm 6.85$ vs. $9.98 \pm 6.40, P=0.0671)$. Serum norepinephrine concentrations in patients with positive PDQ were higher than those in patients with negative PDQ and healthy controls (HC).

$\mathrm{NeP}$ is an important component of AS pain. TNF inhibitors do not appear to be as effective as expected in improving ASassociated NeP. Our result suggested that interference with neurotransmitter activity may be a promising new treatment for AS-associated NeP.

\section{INTRODUCTION}

Ankylosing spondylitis (AS) is an autoinflammatory disease with a largely unknown etiology. It primarily involves the axial skeleton and is characterized by sacroiliac arthritis and enthesitis with pain, stiffness, and joint deformity [1]. The pain in AS patients is currently considered as an inflammatory pain (IP). However, the pain in AS patients is not always correlated with the inflammatory indexes of the disease, such as C-reactive protein (CRP) or the erythrocyte sedimentation rate (ESR) [2]. Biologics are the most effective treatment for AS at present. It was reported that the serological parameters and radiological outcomes were improved in approximately $60 \%$ of AS patients who received tumor necrosis factor (TNF) inhibitors [3]. However, there is a weak correlation between change of inflammation (CRP lever) and back pain in patients with AS after treatment TNF inhibitors [4], while the pain lingers even in the absence of inflammation after administration of the biologics in clinical practice. In 2017, Bidad et al. found that AS-associated pain is not merely attributed to inflammatory pain (IP) but also involves neuropathic pain $(\mathrm{NeP})[2]$.

To date, no large-scale clinical trials have been performed to distinguish IP from NeP. Given the large population of AS patients in China and the heavy economic burden on society and individuals, it is extremely important to find an accurate and practical tool for differentiating between IP and NeP. The painDETECT Questionnaire (PDQ) has proven to be an easily accessible and reliable tool for $\mathrm{NeP}$ screening. The PDQ consists of nine simple selfassessment questions suitable for patient selfscoring and large-scale epidemiological investigation [5].

Our study was to investigate the prevalence and characteristics of NeP in Chinese Han AS patients. We hypothesized that NeP contributed to AS pain with non-inflammatory characteristics.

\section{METHODS}

1. Part one: In the present study, we selected 182 patients with a diagnosis of AS in hospitalization or clinic in our Department of Rheumatology \& Immunology from 
January 2019 to October 2019. The patients who aged $\geq 18$ and diagnosed AS following the 1984 modified New York Criteria [6] were permitted to be enrolled. The patients with fibromyalgia were excluded at enrollment. The patients with fibromyalgia, with a previous diagnosis or under treatment for $\mathrm{NeP}$, previous diagnosis of psychiatric or neurologic conditions, or increased risk to develop neuropathic disorders such as uncontrolled diabetes or neuroendocrine or neurovascular disorders were excluded. Patients using neuropsychiatric drugs or biologics were also excluded (shown in Fig. 1). The general data collected for these patients included age, gender, duration, medication, etc. The Bath Ankylosing Spondylitis Disease Activity Index (BASDAI) [7], the CRP-based Ankylosing Spondylitis Disease Activity Score (ASDAS-CRP) [8], and the BASDAI-Fatigue score were used [9]. NeP was assessed by PDQ [5]. Anxiety and depression levels were determined by using the Hospital Anxiety and Depression Scale (HAD) [10]. Nocturnal pain (NP) and total back pain (TBP) were assessed by a visual analogue scale (VAS). Peripheral pain (PP) was assessed by Q3 of BASDAI.

2. Part two: 105 out of 182 patients started biologics treatment, including etanercept $(n=63)$, infliximab $(n=10)$, adalimumab $(n=28)$ and golimumab $(n=4)$, based on a joint decision by the patient and the rheumatologist [11]. This part of the study included 63 AS patients (31 with PDQ $>12$ and 32 with $P D Q \leq 12$ ) who received a subcutaneous injection of $25 \mathrm{mg}$ etanercept (Enbrel), twice a week for 12 weeks and had no other concomitant medicines. Before administration, all patients underwent strict screening tests for tuberculosis, hepatitis, and tumors. The main clinical parameters included age, gender, ASDAS-CRP, BASDAI-Fatigue, PDQ, and HAD, which were recorded before and after medication administration.

3. Part three: 6 AS patients with PDQ $>19$ and 14 age- and gender-matched patients with PDQ $\leq 19$ after Enbrel treatment were included in this part of study, and 10 ageand gender-matched healthy individuals were used as the controls. Serum

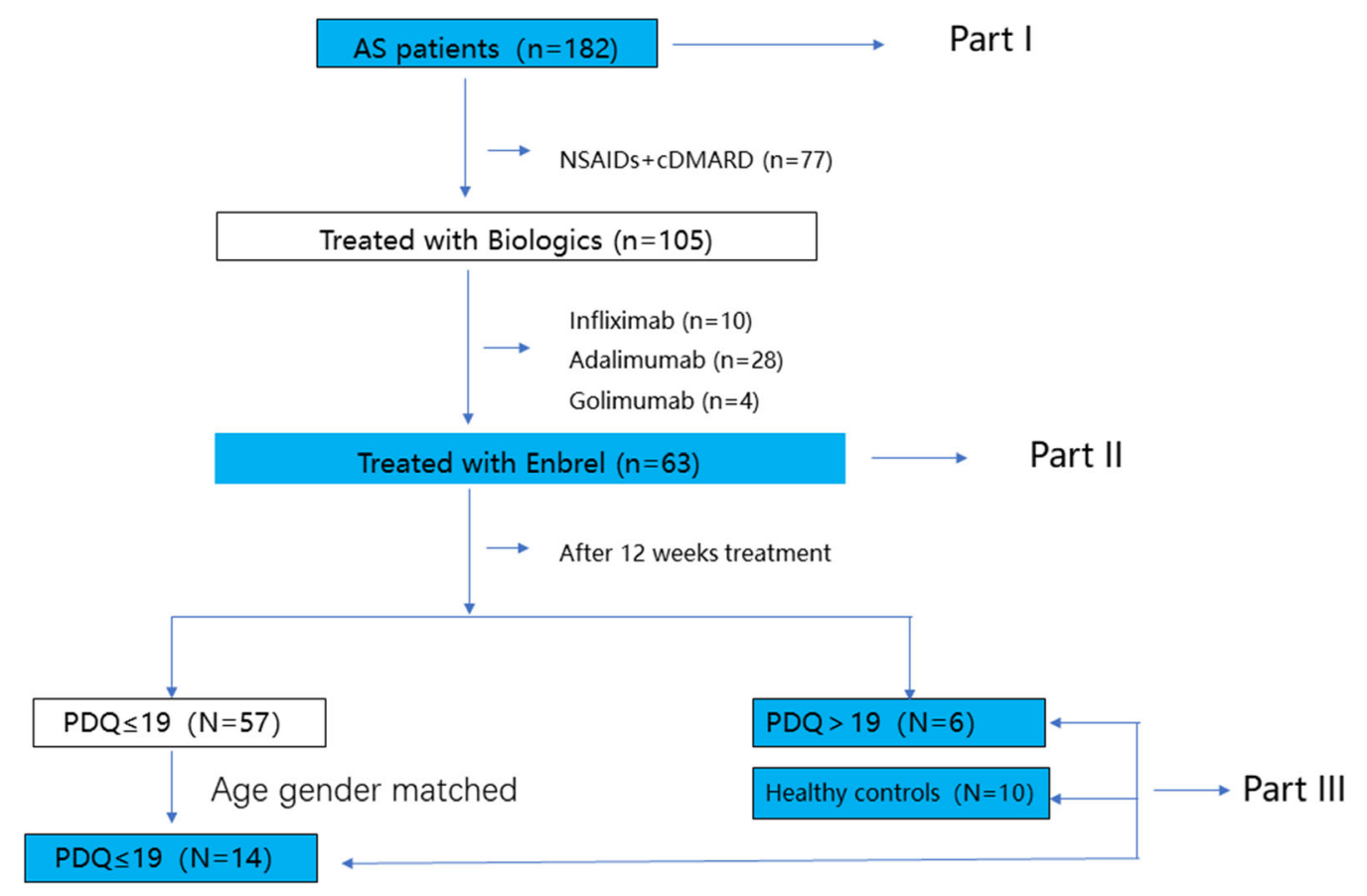

Fig. 1 Flow chart of inclusion and exclusion criteria 
neurotransmitter concentrations, including norepinephrine (NE), 5-hydroxytryptamine (5HT), glycine (Gly), glutamic acid (Glu), $\gamma$ aminobutyric acid (GABA), 5-hydroxyindoleacetic acid (5HIAA), kynurenine $(\mathrm{KYN})$, and tryptophan (TRP), were measured. Differences in the concentrations of these neurotransmitters were compared among the three groups. All blood samples were sent to Anpel Laboratory Technologies (Shanghai, China) for testing.

4. PDQ calculation: The PDQ includes nine simple self-assessment questions with no need for physical examination and therefore is suitable for self-assessment and largescale epidemiological investigation [5]. Of the nine questions, seven are weighted sensory items, which are scored by using a $0-5$ scoring system to indicate severity, and the other two were related to radiating pain and the mode of pain onset. Cut-offs were applied for analysis of the PD-Q as previously described. A total score of nine items $\leq 12$ indicates a Nep is not likely, whereas a score $\geq 19$ indicates that a Nep is likely. Between PD-Q scores of 12 and 19, Nep can be present, but is uncertain.

HAD score: Anxiety and depression were evaluated by using the HAD, in which a score of $0-7$ indicates no anxiety or depression, 8-10 for possible anxiety or depression, and 11-21 for a high probability of both.

The study was conducted according to the Helsinki Declaration and approved by the local ethics committee (ID: 2017SL046). All the patients gave their written informed consent before inclusion in the study.

5. Statistical analyses: Statistical analysis were conducted with SPSS software (ver. 16.0). The data that has a normal distribution are presented as the mean \pm SD. Continuous variables with a normal distribution were evaluated using a two-sample $t$ test. Analysis of variance (ANOVA) was employed to look for multigroup differences, and unordered categorical variables were assessed by the Chi-squared test. Principal component analysis (PCA) was performed by dimension reduction processing with SPSS statistical software. Structural equation modeling (SEM) was analyzed using Amos ver. 23.0.0, and any possible relationships between latent variables were evaluated using SEM.

\section{RESULTS}

1. General clinical information of AS patients

(1) Of the 182 AS patients who participated in the first phase of the study, a total of 14 patients $(7.7 \%)$ had PDQ scores of $\geq 19$ (probable Nep), 55 patients (30.21\%) had PDQ scores rated between 12 and 19 (uncertainty of Nep) and 113 patients $(62.08 \%)$ had PDQ scores $\leq 12$ (unlikely Nep). Cronbach's $\alpha$ coefficient of the PDQ was analyzed to determine the internal consistency of the PDQ in assessing NeP in AS patients. The results showed that the Cronbach's $\alpha$ coefficient of the PDQ in AS patients was 0.92 , suggesting that the PDQ had a relatively high internal consistency and reliability.

(2) There were significant differences in NP (mean \pm standard deviation [SD]: $2.25 \pm 2.85$ vs. $5.34 \pm 4.55$ vs. $5.87 \pm 3.02, \quad P<0.0001)$, TBP (mean \pm SD: $2.23 \pm 2.62$ vs. $5.54 \pm 4.92$ vs. $5.85 \pm 2.48, P<0.0001$ ), and PP (mean \pm SD: $1.25 \pm 1.79$ vs. $2.44 \pm 2.40$ vs. $4.54 \pm 2.54$, $P<0.0001)$ among the three groups (PDQ $\leq 12,12<\mathrm{PDQ}<19$ and $\mathrm{PDQ} \geq 19$ ). There were significant differences in BASDAI, ASDAS, and psychological status, including anxiety, depression, and fatigue, except for CRP (mean \pm SD: $12.75 \pm 18.12$ vs. $14.41 \pm 11.79$ vs. $16.77 \pm 11.36, P=0.6057$ ) (Table 1 ).

2. PCA: Determined by PCA, the first principal component accounted for $62.7 \%$ of the population variance. The first principal component was calculated as follows:

$\mathrm{F} 1=0.881 \times(\mathrm{NP})+0.899 \times(\mathrm{TBP})+0.703$

$\times(\mathrm{NeP})+0.639 \times(\mathrm{PP})$, indicating that the weight of NeP was greater than that of PP in the first principal component (0.703 vs. 0.639$)$. The common factor (CF) of pain was calculated with a rotated component matrix, indicating that there are three major dimensions of pain in AS patients: CF 1 represents NP and TBP, CF 2 represents peripheral pain, and CF 3 represents 
Table 1 Clinical data of AS patients in different groups

\begin{tabular}{llllc}
\hline & PDQ $\leq \mathbf{1 2}(\boldsymbol{n}=\mathbf{1 1 3})$ & $\mathbf{1 2}<\mathbf{P D Q}<\mathbf{1 9}(\boldsymbol{n}=\mathbf{5 5})$ & $\mathbf{P D Q} \geq \mathbf{1 9}(\boldsymbol{n}=\mathbf{1 4})$ & $\boldsymbol{P}$ value \\
\hline Sex $(\mathrm{M} / \mathrm{F})$ & $102 / 11$ & $53 / 2$ & $8 / 6$ & 0.6872 \\
Age (years) & $36.96 \pm 11.60$ & $36.36 \pm 8.88$ & $37.43 \pm 10.78$ & 0.9228 \\
Duration & $10.94 \pm 8.87$ & $8.67 \pm 6.07$ & $14.36 \pm 9.24$ & $0.0458^{*}$ \\
NSARDs & $95 / 113$ & $54 / 55$ & $14 / 14$ & 0.7703 \\
NP & $2.25 \pm 2.85$ & $5.34 \pm 4.55$ & $5.87 \pm 3.02$ & $<0.0001^{* * * *}$ \\
TBP & $2.23 \pm 2.62$ & $5.54 \pm 4.92$ & $5.85 \pm 2.48$ & $<0.0001^{* * * *}$ \\
PP & $1.25 \pm 1.79$ & $2.44 \pm 2.40$ & $4.54 \pm 2.54$ & $<0.0001^{* * * *}$ \\
CRP & $12.75 \pm 18.12$ & $14.41 \pm 11.79$ & $16.77 \pm 11.36$ & 0.6057 \\
ASDAS-CRP & $1.29 \pm 0.76$ & $2.09 \pm 0.70$ & $2.60 \pm 0.81$ & $<0.0001^{* * *}$ \\
BASDAI & $1.71 \pm 1.56$ & $3.72 \pm 1.88$ & $5.16 \pm 1.95$ & $<0.0001^{* * * *}$ \\
HAQ & $4.39 \pm 5.01$ & $8.0 \pm 10.65$ & $8.96 \pm 9.49$ & $0.0033^{* *}$ \\
HAD-A & $4.46 \pm 3.30$ & $6.23 \pm 3.21$ & $7.50 \pm 2.47$ & $0.001^{* * *}$ \\
HAD-D & $5.48 \pm 3.81$ & $6.63 \pm 3.30$ & $9.00 \pm 3.32$ & $0.0016^{*}$ \\
BASDAI-fatigue & $2.58 \pm 2.40$ & $3.87 \pm 2.39$ & $5.67 \pm 2.31$ & $<0.0001^{* * * *}$ \\
\hline
\end{tabular}

$P D Q$ pain detection questionnaire, $N P$ nocturnal pain, $T B P$ total back pain, $P P$ peripheral pain, $C R P C$-reactive protein, $A S D A S$-CRP ankylosing spondylitis disease activity score-CRP, $B A S D A I$ bath assessment of ankylosing spondylitis disease activity index, $H A Q$ health assessment questionnaire, $H A D-A$ hospital anxiety and depression scale-anxiety, $H A D$ $D$ hospital anxiety and depression scale-depression

Data are expressed as the mean \pm standard deviation (SD), $P$ value comparison between the three groups, ${ }^{* * * *} P<0.0001$, ${ }^{* *} P<0.001,{ }^{* *} P<0.01,{ }^{*} P<0.05$

Table 2 Principal component analysis; component matrix

\begin{tabular}{llrlr}
\hline & C1 & \multicolumn{1}{c}{ C 2 } & \multicolumn{1}{c}{ C3 } & \multicolumn{1}{c}{ C 4 } \\
\hline PDQ & 0.703 & 0.422 & -0.572 & 0.001 \\
NP & 0.881 & -0.411 & 0.045 & 0.229 \\
TBP & 0.899 & -0.361 & 0.070 & -0.236 \\
PP & 0.639 & 0.610 & 0.468 & 0.014 \\
\hline
\end{tabular}

Method of extraction: principal component analysis

Four components were extracted

$C$ component, $P D Q$ pain detection questionnaire, $N P$ nocturnal pain, $T B P$ total back pain, $P P$ peripheral pain

NeP. CF analysis showed that $\mathrm{NeP}$ was a relatively independent type of AS pain (Tables 2, 3).
3. Use of SEM: SEM was used to analyze the impact of NeP on disease activity and psychology. BASDAI and ASDAS-CRP were employed to assess the disease activity index, while Hospital Anxiety and Depression Scale-Anxiety (HAD-A) and Hospital Anxiety and Depression Scale-Depression (HAD-D) were used to evaluate the psychological indexes of patients. SEM was successfully established with a probability level of 0.227 , a ratio of Chi-square to degree of freedom (CMIN/DF) of 1.413, a comparative fit index (CFI) of 0.996, a normed fit index (NFI) of 0.987, and a root mean squared error of approximation (RMSEA) of 0.048. The SEM results showed that PDQ could alter disease activity $(\beta=0.62, P<0.001)$, which in turn affected psychological status $(\beta=0.42$, $P<0.001$ ) (Fig. 2). 
Table 3 Principal component analysis; rotated component matrix

\begin{tabular}{lllll}
\hline & C 1 & C 2 & C 3 & C4 \\
\hline PDQ & 0.224 & 0.213 & 0.951 & 0.006 \\
NP & 0.949 & 0.139 & 0.201 & -0.211 \\
TBP & 0.928 & 0.185 & 0.201 & 0.255 \\
PP & 0.176 & 0.963 & 0.205 & 0.006 \\
\hline
\end{tabular}

Method of extraction: principal component analysis

Method of rotation: Caesar normal maximum variance method

Rotation converged in 5 iterations

$P<0.01$ by Bartlett's test; the results of first principal component calculation were as follows: F1 $=0.881 \times($ nocturnal pain $)+0.899 \times($ total back pain $)+0.703 \times($ neuropathic pain $)+0.639 \times($ peripheral pain)

The weight of neuropathic pain was greater than peripheral pain in the first principal component ( 0.703 vs. 0.639$)$. Common factors (CF) of pain calculated by rotated component matrix analysis: CF 1 represents nocturnal pain and low back pain; CF 2 represents peripheral pain; and CF 3 represents PDQ

$C$ component, $P D Q$ pain detection questionnaire, $N P$ nocturnal pain, $T B P$ total back pain, $P P$ peripheral pain

4. Pain improvement after biological treatment: Of the 63 AS patients who used etanercept for 12 weeks, PDQ was $\leq 12$ in 32 patients, and PDQ was $>12$ in the remaining 31 patients. There were no significant differences in sex (male) (mean \pm SD: $26 / 32$ vs. 27/31, $P=0.6059$ ), age (mean \pm SD: $37.97 \pm 12.83$ vs. $36.61 \pm 9.56, P=0.6254$ ), and disease duration (mean $\pm \mathrm{SD}: \quad 12.90 \pm 10.77$ vs. $11.22 \pm 8.79$, $P=0.5592$ ) between two groups. These results suggested that despite the degrees of NP, TBP and PP were significantly decreased after medication administration, PDQ was not significantly decreased (mean \pm SD: $10.60 \pm 6.85$ vs. $9.98 \pm 6.40 \quad P=0.0671$ ). Subgroup analysis revealed that PDQ was decreased significantly in AS patients with $\mathrm{PDQ}>12$ (mean \pm SD: $17.06 \pm 2.40$ vs. $15.55 \pm 3.69, P=0.0059)$ and did not show a significant decrease in AS patients with $\mathrm{PDQ} \leq 12 \quad$ (mean $\pm \mathrm{SD}$ :

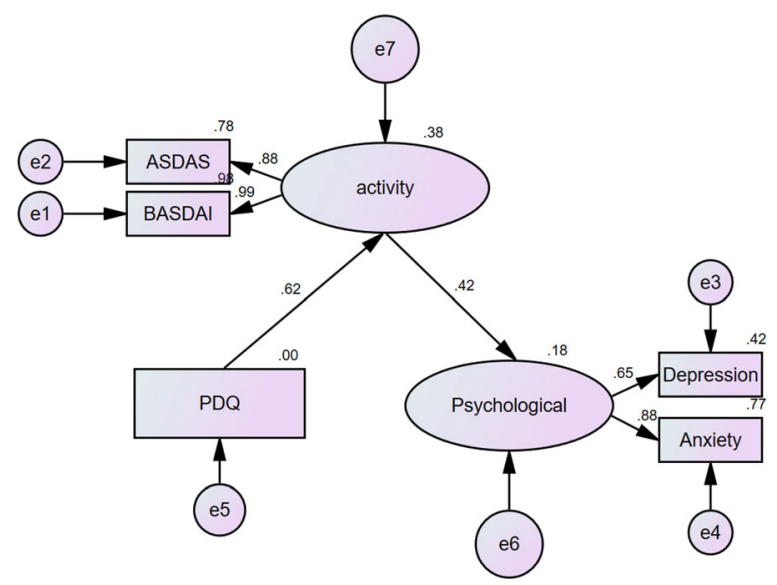

Fig. 2 Structural equation modeling. Structural equation modeling (SEM) on the impact of neuropathic pain on disease activity and psychology. BASDAI and ASDASCRP were used as the disease activity index, and HAD-A and HAD-D were used as the psychological index. SEM was successfully established (probability level $=0.227$; ratio of Chi-square to degree of freedom [CMIN/ $\mathrm{DF}]=1.413 ;$ comparative fit index $[\mathrm{CFI}]=0.996$; normed fit index $[\mathrm{NFI}]=0.987$; and root mean squared error of approximation [RMSEA] $=0.048)$. According to SEM, PDQ could alter the disease activity $(\beta=0.62$, $P<0.001)$, and the disease activity could influence the psychological status $(\beta=0.42, P<0.001)$

\section{$4.34 \pm 2.47 \quad$ vs. $\quad 4.59 \pm 2.79, \quad P=0.5076)$ (Table 4).}

5. Detection of neurotransmitters in AS patients: Of the 20 AS patients in the third part of the study, PDQ was $>19$ in six patients and PDQ $\leq 19$ in 14 patients. The characteristic and clinical data of the patients and healthy controls (HC) are shown in Table 5. The norepinephrine (NE) concentration in patients with PDQ $>19$ was significantly higher than that in patients with $\mathrm{PDQ} \leq 19$ and $\mathrm{HC}(0.21 \pm 0.06$ vs. $0.13 \pm 0.05$ vs. $0.07 \pm 0.02, \quad P<0.001)$. There was no significant difference in other neurotransmitter concentrations.

\section{DISCUSSION}

Our research indicates that AS-associated pain was not merely a kind of IP but also involved NeP. TNF inhibitors are seemingly ineffective at improving AS-associated NeP. The change in NE 


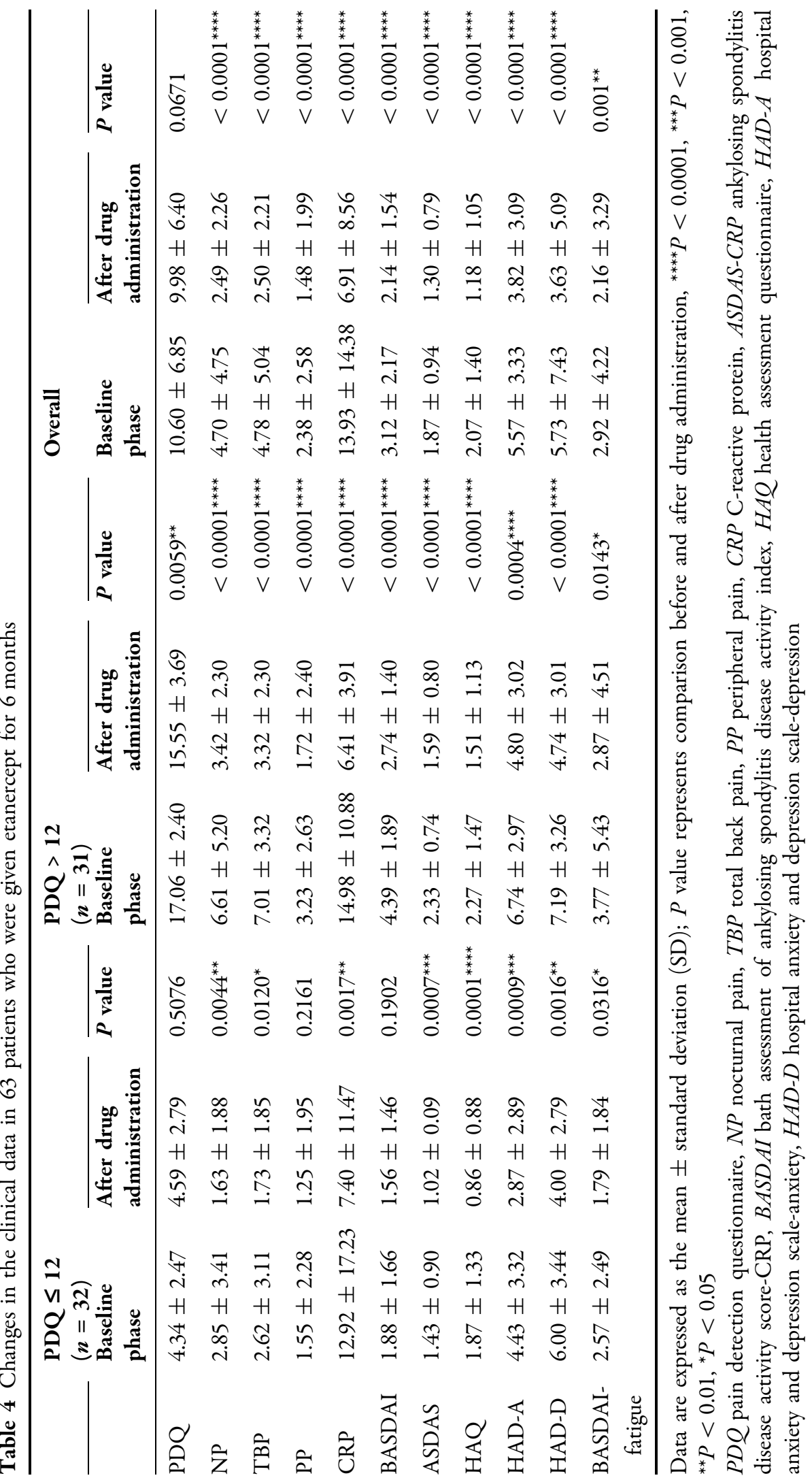


Table 5 Changes of neurotransmitter concentrations in peripheral blood of 20 AS patients and ten healthy controls

\begin{tabular}{lllll}
\hline & PDQ $>\mathbf{1 9}(\boldsymbol{n}=\mathbf{6})$ & PDQ $\leq \mathbf{1 9}(\boldsymbol{n}=\mathbf{1 4})$ & Control $(\boldsymbol{n}=\mathbf{1 0})$ & $\boldsymbol{P}$ \\
\hline Sex $(\mathrm{M} / \mathrm{F})$ & $4 / 2$ & $13 / 1$ & $8 / 2$ & 0.3338 \\
Age & $42.67 \pm 4.27$ & $36.57 \pm 2.72$ & $0.21 \pm 0.06$ & 0.2394 \\
Duration & $18.67 \pm 2.47$ & $11.94 \pm 2.19$ & & 0.0887 \\
CRP & $5.02 \pm 2.60$ & $8.09 \pm 6.79$ & & 0.3028 \\
BASDAI & $4.27 \pm 1.89$ & $2.15 \pm 1.17$ & & $0.0064^{* *}$ \\
NE & $0.21 \pm 0.06$ & $0.13 \pm 0.05$ & $0.07 \pm 0.02$ & $<0.001^{* * *}$ \\
5HT & $22.62 \pm 9.65$ & $20.50 \pm 17.85$ & $19.96 \pm 10.01$ & 0.9338 \\
Gly & $110.8 \pm 21.37$ & $96.61 \pm 25.24$ & $95.32 \pm 34.52$ & 0.5223 \\
Glu & $16.64 \pm 7.32$ & $29.83 \pm 29.05$ & $19.66 \pm 5.05$ & 0.3277 \\
GABA & $587.7 \pm 19.01$ & $629.0 \pm 336.0$ & $523.4 \pm 135.4$ & 0.6213 \\
5HIAA & $2.21 \pm 0.97$ & $3.97 \pm 3.87$ & $2.62 \pm 0.67$ & 0.3277 \\
KYN & $78.35 \pm 25.35$ & $83.87 \pm 44.80$ & $69.79 \pm 18.05$ & 0.4845 \\
TRP & $1,860 \pm 402.7$ & $2,067 \pm 482.0$ & $2,008 \pm 446.7$ & 0.6528 \\
\hline
\end{tabular}

Data are expressed as the mean \pm standard deviation (SD); $P$ value represents comparison between the three groups, ${ }^{* * *} P<0.001,{ }^{* *} P<0.01,{ }^{*} P<0.05$

CRP C-reactive protein, BASDAI bath assessment of ankylosing spondylitis disease activity index, $N E$ norepinephrine, $5 H T$ serotonin, Gly glycine, Glu glutamic acid, GABA $\gamma$-aminobutyric acid, SHIAA 5-hydroxyindoleacetic acid, KYN kynurenine, TRP tryptophan

concentrations in the peripheral blood of AS patients with $\mathrm{NeP}$ suggests that interference with neurotransmitter actions is a promising new approach to the treatment of AS-associated $\mathrm{NeP}$.

The main symptom of AS is pain, which is currently recognized as one type of IP [12]. Thus, previous studies and the development of new anti-AS medications have mainly focused on blocking inflammatory factors [13], [14], [15]. Given the large number of outpatients and lack of communication time between physicians and patients in China and the actual clinical situation in China, both physicians and patients usually pay attention to laboratory testing and imaging findings, inevitably leading to overlooking pain and other subjective symptoms. However, it has been commonly observed that some AS patients complain of pain even when inflammatory indexes such as ESR and CRP are within the normal ranges or biologics have been administered. It seems incomplete and biased to entirely attribute the clinical symptoms of AS to IP. Increased studies in recent years have found that pain in AS patients is not always correlated with inflammation [16], [17]. In addition, this kind of pain often has the characteristics of $\mathrm{NeP}$, including numbness and pricking sensations. Freynhagen et al. investigated more than 8000 patients with chronic pain and found that approximately one-third had pain of a neuropathic nature [18]. Using head MRI, Wu et al. demonstrated NeP in AS patients and revealed that the corresponding abnormality in the grey matter was correlated with clinical symptoms [19].

In the clinic, fibromyalgia is a neuropathic pain syndrome and is common in patients with histories of inflammatory diseases with symptoms of chronic pain, including Sjögren's syndrome, systemic lupus erythematosus (SLE), or rheumatoid arthritis (RA). However, for AS 
patients, the evidence is lacking and controversial [20]. Previous studies have reported the high prevalence of fibromyalgia in patients with AS [21], [22]. In contrast, a recent study stated that compared with normal people, the incidence of fibromyalgia did not increase in patients with AS [23]. In the Chinese population, so far, there is no epidemiological data on fibromyalgia syndrome. As a neuropathic pain (Nep) syndrome, fibromyalgia contributes to multifocal pain. Because of the dysregulated function in the central nervous system and amplification of the sense of pain, symptoms such as musculoskeletal pain, visceral pain, and chronic headaches are often observed in patients [24]. In fibromyalgia patients, changes in the number of inflammatory cytokines have been widely reported, and the role of neurogenic inflammation has been thought to play a potential role between inflammatory disorder and the development of fibromyalgia [25]. Therefore, the patients with fibromyalgia were excluded at enrollment to reduce the bias in this study. Despite exclusion of fibromyalgia, a total of 14 patients (7.7\%) still showed probable Nep with PDQ scores of $\geq 19$, which further confirmed the important role of Nep in the pain of AS patients and needed attention in clinical.

Diagnosis of a neuropathic component is challenging because there is currently no universally accepted gold standard. Previous studies have confirmed that the PainDETECT questionnaire (PDQ), Douleur Neuropathique en 4 Questions (DN4), and Leeds Assessment of Neuropathic Symptoms and Signs (LANSS) are all reliable screening tools for neuropathic pain (Nep) and have been validated for the use in populations with NeP. All of these questionnaire tools have also been validated as self-report items and are therefore suitable for use in a postal/online survey research [5], [26]. Among the three questionnaires, the PDQ was translated into and validated in multiple languages. PDQ is nowadays used for clinical and research purposes [27]. A study in Chinese Nep populations showed that the Chinese version of PDQ scale had a good retest reliability and validity (intraclass correction coefficient $[$ ICC $]=0.973$ ), which can be applied to the clinical screening of Chinese patients with Nep [28]. The PDQ is self- administered questionnaire, specifically developed and validated for use in patients with low back pain with sensitivity and specificity of 85 and $80 \%$, respectively, with a positive predictive value of $83 \%$ [5]. Cronbach's $\alpha$ coefficient of the PDQ was analyzed to determine the internal consistency of the Chinese PDQ in assessing fatigue in AS patients. The coefficient of the PDQ in AS was 0.92, suggesting that the Chinese PDQ had a relatively high internal consistency and reliability. It is feasible to apply a Chinese PDQ in practice to assess neuropathic pain components.

Despite the importance of $\mathrm{NeP}$ in $\mathrm{AS}$ patients, the prevalence and characteristics of $\mathrm{NeP}$ components in patients with AS in clinical practice is limited. The present study contributes to fill this gap. In the present study, approximately one-third of 182 AS patients had likely or uncertain NeP. We extrapolate that the Nep component in AS is underdiagnosed, underestimated in importance, and undertreated. Borman et al. used the LANSS and DN4 to assess pain in 58 AS patients and found that more than 50\% had NeP [29]. Choi et al. used the PDQ to assess 105 AS patients and reported that $15(14.2 \%)$ had NeP. All of these findings suggest that NeP commonly occurs in AS patients [30]. In addition, the PCA of pain in our study showed that the weight of $\mathrm{NeP}$ was greater than that of PP. CF analysis demonstrated that pain in AS consists of three relatively independent dimensions, which are represented by IP (NP and TBP), NeP, and PP. This finding suggests that NeP is an important component of AS pain. The SEM results also suggested that $\mathrm{NeP}$ affects the disease severity of AS, which can further alter the psychological status of AS patients. In summary, these findings indicate that the incidence of $\mathrm{NeP}$ in $\mathrm{AS}$ patients is much higher than we expected before and $\mathrm{NeP}$ plays a critical role in AS disease severity, thereby further affecting the psychological status of AS patients.

Studies on the therapeutic effect of TNF- $\alpha$ on $\mathrm{NeP}$ have revealed meaningful results. In the past, some studies have reported that local or spinal administration of medication that antagonize TNF- $\alpha$ activity attenuates pain in neuropathic animal models [31], [32]. Other 
studies found that mechanical allodynia in a rat model of central neuropathic pain, produced by T13 spinal cord hemisection, was attenuated by the immediate intrathecal administration of etanercept 1-4 weeks after injury to the spinal cord [33]. To the best of our knowledge, however, we are unaware of any randomized controlled clinical trials of infliximab or etanercept for the treatment of other forms of NeP. TNF inhibitors are currently the most effective medication for AS. Our study demonstrated that axial pain, $\mathrm{PP}$, and various inflammatory indexes were improved significantly in AS patients who were administered etanercept for 12 weeks, whereas PDQ was not improved statistically, suggesting that NeP poses a particular treatment challenge to physicians. At present, the therapeutic field of AS is developing rapidly and there are also biologic medicines such as interleukin-17 (IL-17) and Janus kinase (JAK) inhibitors. It was reported that interleukin-17 (IL-17) can regulate neuron-glial communications, synaptic transmission, and Nep after chemotherapy [34]. AG490 (tyrosine kinase inhibitors) is effective for reducing oxaliplatininduced acute Nep. This new function of AG490 is anti-neuroinflammatory and probably acts by inhibiting the JAK/STAT3 signaling; however, the detailed and precise mechanism still needs further investigation [35].

In order to better understand the pathogenesis of Nep in AS patients, we further consulted the related literature. Several studies have reported that the various endogenous transmitters (noradrenaline, dopamine, glutamate, gamma-aminobutyric acid [GABA], serotonin, met- and leu-enkephalins, $\beta$-endorphin, dynorphins, cannabinoids, adenosine triphosphate [ATP]) and various receptors $(\alpha 2, \mu$, etc.) have a close correlation with neuropathic pain [36], [37]. We detected eight neurotransmitters in the peripheral blood of 20 AS patients and found that the serum NE concentrations in AS patients with higher PDQ were significantly elevated. Both NE and 5HT are pain-related neurotransmitters that participate in regulating both algesic and analgesic processes. Peripherally, these neurotransmitters are algesic factors that exert a regulatory effect on local neurocytes via second messengers such as calcium ions or cyclic nucleotides or through a paracrine pathway producing pain by the stimulation of sensory nerve endings. Detection of the concentration of algesic factors in peripheral blood can, to some extent, reflect the degree of pain [36]. Based on this finding, we are using pregabalin to treat $\mathrm{NeP}$ in AS patients, and the related data are still being collected.

There are some limitations in this study. Firstly, the sample size was relatively small. Secondly, we only detected neurotransmitters in the peripheral blood of a limited number of AS patients. We intend to further expand the sample size in our ongoing studies and use head MRI to investigate the structural or functional changes in the pain-related regions of the central nervous system.

\section{CONCLUSIONS}

In summary, $\mathrm{NeP}$ is a common occurrence in $\mathrm{AS}$ patients. It is an important component and one of the three dimensions of AS pain. TNF inhibitors do not appear to be as effective as expected in improving AS-associated NeP. The NE concentrations in the peripheral blood of AS patients with NeP complications are significantly elevated, suggesting that interference with neurotransmitter activity may be a promising new treatment for AS-associated NeP.

\section{Declarations}

Funding The study and the journal's Rapid Service Fee were supported by the National Natural Science Foundation of China (Grant No. 31821003), National Key Research and Development Project (Grant No. 2018AAA0100302), Shanghai Municipal Key Clinical Specialty (shslczdzk02602), and Shanghai Science and Technology Development Funds (2020-SH-XY-2).

Authorship All named authors meet the International Committee of Medical Journal Editors (ICMJE) criteria for authorship for this article, take responsibility for the integrity of the work as a whole, and have given their approval for this version to be published. 
Authorship Contributions Conception and design of the study: HJX, LZ and TL. Acquisition, analysis and interpretation of data: XW, HJL, LL, LYY, JY, JZ, and XWW. Drafting the article and revising it critically for important intellectual content: LZ, TL, XW, HJL, and HJX. Final approval of the article: LZ, TL, XW, HJL, LL, LLY, JY, JZ, XWW, JYB, and HJX.

Disclosures Ling Zhou, Ting Li, Xin Wu, Hongjuan Lu, Li Lin, Lingying Ye, Jian Yin, Juan Zhao, Xiuwen Wang, Jianye Bian, and Huji Xu declare that they have nothing to disclose.

Compliance with Ethics Guidelines The study was conducted according to the Helsinki Declaration and approved by the ethics community of Shanghai Changzheng Hospital (ID: 2017SL046). All patients gave written informed consent. Participants included in our study provided consent for publication if any identifying information is included in the manuscript.

Data Availability The datasets generated during and/or analyzed during the current study are available from the corresponding author on reasonable request. We thank all the participants participating in our research.

\section{OPEN ACCESS}

This article is licensed under a Creative Commons Attribution-NonCommercial 4.0 International License, which permits any noncommercial use, sharing, adaptation, distribution and reproduction in any medium or format, as long as you give appropriate credit to the original author(s) and the source, provide a link to the Creative Commons licence, and indicate if changes were made. The images or other third party material in this article are included in the article's Creative Commons licence, unless indicated otherwise in a credit line to the material. If material is not included in the article's Creative Commons licence and your intended use is not permitted by statutory regulation or exceeds the permitted use, you will need to obtain permission directly from the copyright holder. To view a copy of this licence, visit http://creativecommons.org/licenses/bync/4.0/.

\section{REFERENCES}

1. Zong HX, Xu SQ, Tong H, Wang XR, Pan MJ, Teng YZ. Effect of anti-tumor necrosis factor alpha treatment on radiographic progression in patient with ankylosing spondylitis: a systematic review and meta-analysis. Mod Rheumatol. 2019;29(3): 503-9.

2. Bidad K, Gracey E, Hemington KS, Mapplebeck JCS, Davis KD, Inman RD. Pain in ankylosing spondylitis: a neuro-immune collaboration. Nat Rev Rheumatol. 2017;13(7):410-20.

3. Braun J, van der Horst-Bruinsma IE, Huang F, et al. Clinical efficacy and safety of etanercept versus sulfasalazine in patients with ankylosing spondylitis: a randomized, double-blind trial. Arthritis Rheum. 2011;63(6):1543-51.

4. Hammoudeh M, Zack DJ, Li W, Stewart VM, Koenig AS. Associations between inflammation, nocturnal back pain and fatigue in ankylosing spondylitis and improvements with etanercept therapy. J Int Med Res. 2013;41(4):1150-9.

5. Freynhagen R, Baron R, Gockel U, Tolle TR. painDETECT: a new screening questionnaire to identify neuropathic components in patients with back pain. Curr Med Res Opin. 2006;22(10):1911-20.

6. Goie The HS, Steven MM, van der Linden SM, Cats A. Evaluation of diagnostic criteria for ankylosing spondylitis: a comparison of the Rome, New York and modified New York criteria in patients with a positive clinical history screening test for ankylosing spondylitis. Br J Rheumatol. 1985;24(3):242-9.

7. Dernis-Labous E, Messow M, Dougados M. Assessment of fatigue in the management of patients with ankylosing spondylitis. Rheumatology (Oxford). 2003;42(12):1523-8.

8. van der Heijde D, Lie E, Kvien TK, et al. ASDAS, a highly discriminatory ASAS-endorsed disease activity score in patients with ankylosing spondylitis. Ann Rheum Dis. 2009;68(12):1811-8.

9. van Tubergen A, Coenen J, Landewe $\mathrm{R}$, et al. Assessment of fatigue in patients with ankylosing spondylitis: a psychometric analysis. Arthritis Rheum. 2002;47(1):8-16. 
10. Zigmond AS, Snaith RP. The hospital anxiety and depression scale. Acta Psychiatr Scand. 1983;67(6): 361-70.

11. van der Heijde D, Ramiro S, Landewé R, et al. 2016 update of the ASAS-EULAR management recommendations for axial spondyloarthritis. Ann Rheum Dis. 2017;76(6):978-91.

12. Lindstrom U, Bremander A, Haglund E, Bergman S, Petersson IF, Jacobsson LT. Back pain and health status in patients with clinically diagnosed ankylosing spondylitis, psoriatic arthritis and other spondyloarthritis: a cross-sectional populationbased study. BMC Musculoskelet Disord. 2016;17: 106.

13. Mease P, Walsh JA, Baraliakos X, et al. Translating improvements with ixekizumab in clinical trial outcomes into clinical practice: ASAS40, pain, fatigue, and sleep in ankylosing spondylitis. Rheumatol Ther. 2019;6(3):435-50.

14. Deodhar A, Conaghan PG, Kvien TK, et al. Secukinumab provides rapid and persistent relief in pain and fatigue symptoms in patients with ankylosing spondylitis irrespective of baseline C-reactive protein levels or prior tumour necrosis factor inhibitor therapy: 2-year data from the MEASURE 2 study. Clin Exp Rheumatol. 2019;37(2):260-9.

15. Benucci M, Damiani A, Li Gobbi F, et al. Therapeutic potential of ixekizumab in the treatment of ankylosing spondylitis: a review on the emerging clinical data. Ther Clin Risk Manag. 2020;16: 287-97.

16. Li T, Liu Y, Sheng R, Yin J, Wu X, Xu H. Correlation between chronic pain acceptance and clinical variables in ankylosing spondylitis and its prediction role for biologics treatment. Front Med (Lausanne). 2020;7:17.

17. Kim TW, Son SM, Lee JS. Neuropathic pain in ankylosing spondylitis: a meta-analysis. Z Rheumatol. 2020;79(1):95-102.

18. Freynhagen R, Baron R, Tolle T, et al. Screening of neuropathic pain components in patients with chronic back pain associated with nerve root compression: a prospective observational pilot study (MIPORT). Curr Med Res Opin. 2006;22(3):529-37.

19. Wu Q, Inman RD, Davis KD. Neuropathic pain in ankylosing spondylitis: a psychophysics and brain imaging study. Arthritis Rheum. 2013;65(6): 1494-503.

20. Gau SY, Lee YH, Tsou HK, et al. Patients with ankylosing spondylitis are associated with high risk of fibromyalgia: a nationwide population-based cohort study. Front Med (Lausanne). 2021;8: 618594.

21. Almodovar R, Carmona L, Zarco $\mathrm{P}$, et al. Fibromyalgia in patients with ankylosing spondylitis: prevalence and utility of the measures of activity, function and radiological damage. Clin Exp Rheumatol. 2010;28(6 Suppl 63):S33-9.

22. Ablin JN, Eshed I, Berman M, et al. Prevalence of axial spondyloarthritis among patients with fibromyalgia: a magnetic resonance imaging study with application of the assessment of spondyloarthritis international society classification criteria. Arthritis Care Res (Hoboken). 2017;69(5):724-9.

23. Sayin S, Yurdakul FG, Sivas F, Bodur H. Is fibromyalgia frequency increasing in axial spondyloarthritis? Association with fibromyalgia and biological therapies. Rheumatol Int. 2020;40(11): 1835-41.

24. Clauw DJ. Fibromyalgia: a clinical review. JAMA. 2014;311(15):1547-55.

25. Ablin J, Neumann L, Buskila D. Pathogenesis of fibromyalgia - a review. Joint Bone Spine. 2008;75(3):273-9.

26. Bailly F, Cantagrel A, Bertin P, et al. Part of pain labelled neuropathic in rheumatic disease might be rather nociplastic. RMD Open. 2020;6(2).

27. Freynhagen R, Tolle TR, Gockel U, Baron R. The painDETECT project-far more than a screening tool on neuropathic pain. Curr Med Res Opin. 2016;32(6):1033-57.

28. Zhao C, Chen C, Xiong S. The preliminary probe of reliability and validity of Chinese PDQ scale in evaluation of neuropathic pain. In The 18th national neurology academic conference of Chinese Medical Association. 2015.

29. Borman P, Kaygisiz F, Yaman A. Neuropathic component of low back pain in patients with ankylosing spondylitis. Mod Rheumatol. 2020;2020:1-6.

30. Choi JH, Lee SH, Kim HR, Lee KA. Association of neuropathic-like pain characteristics with clinical and radiographic features in patients with ankylosing spondylitis. Clin Rheumatol. 2018;37(11): 3077-86.

31. Svensson CI, Schafers M, Jones TL, Powell H, Sorkin LS. Spinal blockade of TNF blocks spinal nerve ligation-induced increases in spinal P-p38. Neurosci Lett. 2005;379(3):209-13.

32. Andrade P, Hoogland G, Del Rosario JS, Steinbusch HW, Visser-Vandewalle V, Daemen MA. Tumor necrosis factor-alpha inhibitors alleviation of 
experimentally induced neuropathic pain is associated with modulation of TNF receptor expression. J Neurosci Res. 2014;92(11):1490-8.

33. Marchand F, Tsantoulas C, Singh D, et al. Effects of etanercept and minocycline in a rat model of spinal cord injury. Eur J Pain. 2009;13(7):673-81.

34. Luo H, Liu HZ, Zhang WW, et al. Interleukin-17 regulates neuron-glial communications, synaptic transmission, and neuropathic pain after chemotherapy. Cell Rep. 2019;29(8):2384-97.
35. Li SF, Ouyang BS, Zhao X, Wang YP. Analgesic effect of AG490, a Janus kinase inhibitor, on oxaliplatin-induced acute neuropathic pain. Neural Regen Res. 2018;13(8):1471-6.

36. Ban EG, Brassai A, Vizi ES. The role of the endogenous neurotransmitters associated with neuropathic pain and in the opioid crisis: the innate painrelieving system. Brain Res Bull. 2020;155:129-36.

37. Sisignano M, Lotsch J, Parnham MJ, Geisslinger G. Potential biomarkers for persistent and neuropathic pain therapy. Pharmacol Ther. 2019;199:16-29. 